\title{
Anisotropic and mass-dependent energization of heavy ions by kinetic Alfvén waves
}

\author{
D. J. $\mathrm{Wu}^{1}$ and L. Yang ${ }^{1,2}$ \\ 1 Purple Mountain Observatory, 2 West Beijing Road, Nanjing 210008, PR China \\ e-mail: djwu@pmo.ac.cn \\ 2 Graduate School of Chinese Academy of Sciences, Beijing 100039, PR China
}

Received 13 March 2006 / Accepted 13 April 2006

\begin{abstract}
Context. Some recent observations of the solar corona suggest that the heavy ions undergo an anisotropic (mainly across the magnetic field) and mass-dependent energization.

Aims. In this work, we investigate the nonlinear interaction of heavy ions with kinetic Alfvén waves, in particular, their energization in the nonlinear kinetic Alfvén waves.

Methods. Based on a three-component plasma model including electrons, protons, and heavy ions, a localized nonlinear structure of kinetic Alfvén wave is presented. In the nonlinear wave, the heavy ions are energized in the cross-field and field-aligned by the perpendicular polarization and the parallel acceleration of the wave electric field.

Results. It is found that the ion cross-field energization depends on its mass and abundance in the same way that the velocity is proportional to the mass-charge ratio and decreases with the abundance ratio. On the other hand, the ion field-aligned energy is proportional to the charge, but is free of the mass and abundance. In the low abundance case $(<10 \%)$, in particular, the minor heavy ions have strongly anisotropic temperature as well as are hotter and flow faster than protons. The resulting ion-proton temperature ratio is proportional to the mass number and the square of the mass-charge ratio, and the ion perpendicular-parallel temperature ratio is proportional to the cube of the mass-charge ratio.

Conclusions. This anisotropic and mass-dependent energization mechanism by kinetic Alfvén waves for ions can be potentially important for understanding the microphysics of the energization of minor heavy ions observed in the solar corona.
\end{abstract}

Key words. acceleration of particles - plasmas - waves - Sun: corona

\section{Introduction}

Remote observations with the UVCS (Ultraviolet Coronagraph Spectrometer) on board SOHO (Solar and Heliospheric Observatory) show that heavy ions such as $\mathrm{O}^{5+}$ and $\mathrm{Mg}^{9+}$ have experienced preferential cross-field heating, causing strong temperature anisotropy $T_{\perp} / T_{\|}>10$, and are hotter and flow faster than protons in the inner heliosphere (Kohl et al. 1997; Li et al. 1998; Esser et al. 1999; Cranmer et al. 1999; Marsch \& Tu 2001). A popular explanation for the ion energization across the ambient magnetic field is based on the ion-cyclotron damping of high-frequency Alfvén waves injected into the base of the solar corona (Marsch \& Tu 2001; Ofman et al. 2002; Hong et al. 2004), in which the wave dissipation range is formed by the high-frequency waves with frequencies close to the local ion gyrofrequency (Hollweg \& Isenberg 2002). The ioncyclotron heating scheme, however, is not free of difficulty (Voitenko \& Goossens 2004a, 2005a,b). Recently, in situ observations from the Cluster and FAST spacecrafts show that the low-frequency dispersive Alfvén waves with short cross-field wavelengths, called Kinetic Alfvén Wave (KAW), can be responsible for the cross-field energization of ions, as well as for the field-aligned energization of electrons that drive bright aurora (Wygant et al. 2002; Chaston et al. 2003, 2004, 2005). On the other hand, recently theoretical work also shows that KAWs can efficiently produce the both electron heating in bright coronal loops (Wu \& Fang 1999) and in dense coronal plumes
(Wu \& Fang 2003) and acceleration in auroral plasmas (Wu \& Chao 2003, 2004a).

This KAW can be created when an obliquely propagating Alfvén wave is affected by the electron temperature $\left(k \rho_{\mathrm{s}} \sim 1\right.$ for $\alpha>1)$ and inertia $\left(k \lambda_{\mathrm{e}} \sim 1\right.$ for $\left.\alpha<1\right)$, where $k=2 \pi / \lambda$ is the wavenumber, $\alpha=v_{T_{\mathrm{e}}} / v_{\mathrm{A}}$ the ratio of the electron thermal speed $v_{T_{\mathrm{e}}}$ to the Alfvén speed $v_{\mathrm{A}}, \rho_{\mathrm{s}}=\sqrt{Q} v_{T_{\mathrm{e}}} / \omega_{\mathrm{cp}}$ the ion acoustic gyroradius, $\lambda_{\mathrm{e}}=\sqrt{Q} v_{\mathrm{A}} / \omega_{\mathrm{cp}}$ the electron inertial length, $Q=m_{\mathrm{e}} / m_{\mathrm{p}}$ the mass ratio of electron to proton, and $\omega_{\mathrm{cp}}$ the proton gyrofrequency (Hasegawa \& Uberoi 1982). Since the pioneering theoretical work of Hasegawa \& Chen (1975), KAW has been an increasingly interesting topic in both experimental and theoretical studies. In the experimental, KAW has been observed both in space (Louarn et al. 1994; Chaston et al. 1999) and the laboratory (Leneman et al. 1999; Kletzing et al. 2003) plasmas. Also, theories of linear and nonlinear KAW in electron-proton plasmas have been studied extensively and been applied to various fields from laboratory to space and astrophysical plasmas, since the parallel component of its electric field $E_{\|}$can play an important role in the particle energization of magnetized plasmas (see e.g. Wu \& Fang 1999, 2003; Wu \& Chao 2003, 2004a,b; Stasiewicz et al. 2000).

In a recent work based on a three-component (electrons, protons, and heavy ions) plasma model, we studied the interaction of heavy ions with linear KAWs (Yang \& Wu 2005). In this letter, we go on to investigate the nonlinear interaction of heavy 
ions with KAWs based on the three-component model, in particular, the energization of minor heavy ions in nonlinear KAWs. The result shows that the ion cross-field energy (or i.e. temperature) is proportional to its mass number and the square of its mass-charge ratio and decreases with its background abundance ratio. On the other hand, the ion field-aligned energy is proportional to its charge, but free of its mass and abundance. In consequence, the heavy ions have strongly anisotropic temperature as well as are hotter and flow faster than protons. We anticipate that this novel energization mechanism is potentially important for explaining observations of the anisotropic and mass-dependent energization of minor heavy ions in the solar corona.

\section{Nonlinear interaction of heavy ions with KAW}

In a three-component plasma (electrons, protons, and heavy ions) magnetized by a magnetic field $\boldsymbol{B}_{0}$ along the $z$ axis, the equation set governing the dynamics of KAWs consists of the following (Wu et al. 1995, 1996; Wu \& Chao 2004b): (i) the continuity equations of electrons, protons, and heavy ions

$\begin{aligned} d_{\eta}\left[n_{\mathrm{e}}\left(k_{z} v_{\mathrm{e} z}-M\right)\right] & =0, \\ d_{\eta}\left[n_{\mathrm{p}}\left(k_{x} v_{\mathrm{p} x}-M\right)\right] & =0, \\ d_{\eta}\left[n_{\mathrm{i}}\left(k_{x} v_{\mathrm{i} x}-M\right)\right] & =0 ;\end{aligned}$

(ii) the parallel momentum equation of electrons

$E_{z}=\left(M-k_{z} v_{\mathrm{e} z}\right) d_{\eta} v_{\mathrm{e} z}-\alpha k_{z} d_{\eta} \ln n_{\mathrm{e}} ;$

(iii) the electric polarization drifts for protons and ions

$v_{\mathrm{p} x}=-M d_{\eta} E_{x}, \quad v_{\mathrm{i} x}=-A_{\mathrm{i}} M d_{\eta} E_{x}=A_{\mathrm{i}} v_{\mathrm{p} x} ;$

(iv) the Faraday's and Ampere's laws for the electromagnetic field

$d_{\eta}\left(k_{z} E_{x}-k_{x} E_{z}\right)=M d_{\eta} B_{y} ; \quad k_{x} d_{\eta} B_{y}=-n_{\mathrm{e}} v_{\mathrm{e} z} ;$

and (v) the charge neutrality condition

$n_{\mathrm{e}}=n_{\mathrm{p}}+Z_{\mathrm{i}} n_{\mathrm{i}}$,

where $\left(k_{x}, 0, k_{z}\right)$ is the unit wave vector, $M=\omega / k$ the phase speed, $\omega$ the frequency, $A_{\mathrm{i}}=m_{\mathrm{i}} /\left(m_{\mathrm{p}} Z_{\mathrm{i}}\right)$ the ion mass-charge ratio, $Z_{\mathrm{i}}=q_{\mathrm{i}} / e$ the ion ionized time number, $\eta=k_{x} x+k_{z} z-M t$ the dimensionless phase parameter along the wave propagating direction, and $d_{\eta}$ denotes the derivative with respect to $\eta$. In the above equation set, space $(x, y, z)$, time $t$, particle number densities $n_{\mathrm{e}(p, i)}$, velocities $\boldsymbol{v}_{\mathrm{e}(p, i)}$, electric field $E_{x(z)}$, and magnetic field $B_{y}$ have been normalized by the electron inertial length $\lambda_{\mathrm{e}}$, the electron Alfvén time $\lambda_{\mathrm{e}} / v_{\mathrm{A}}=\sqrt{Q} / \omega_{\mathrm{ci}}$, the unperturbed electron density $n_{0}$, the Alfvén velocity $v_{\mathrm{A}}=B_{0} / \sqrt{\mu_{0} n_{0} m_{\mathrm{p}}}, \sqrt{Q} v_{\mathrm{A}} B_{0}$, and $\sqrt{Q} B_{0}$, respectively.

Integrating the above equation set by using the localized boundary conditions for a solution with a localized solitary structure: $n_{\mathrm{e}}=1, n_{\mathrm{p}}=n_{\mathrm{p} 0}=1-Z_{\mathrm{i}} n_{\mathrm{i} 0}, n_{\mathrm{i}}=n_{\mathrm{i} 0}, v_{\mathrm{e} z}=v_{\mathrm{p}(\mathrm{i}) x}=$ $d_{\eta}=0$ for $\eta \rightarrow \pm \infty$, where $n_{\mathrm{i} 0}$ is the ion abundance ratio in the background plasma (i.e. the unperturbed ion number density in units of the unperturbed electron density). Limiting the attention on the low- $\beta$ case of $\alpha \ll 1$, one can obtain the nonlinear equation of the electron density in the nonlinear KAW as follows (Wu et al. 1995; Wu \& Chao 2004b):

$\left(d_{\eta} n_{\mathrm{e}}\right)^{2} / 2+K\left(n_{\mathrm{e}}\right)=0$, where

$$
K\left(n_{\mathrm{e}}\right)=\frac{n_{\mathrm{e}}^{6}}{k_{x}^{2} M_{z}^{2}}\left[\frac{\left(n_{\mathrm{e}}-1\right)^{2}\left(2+n_{\mathrm{e}}-3 M_{z}^{2} n_{\mathrm{e}}\right)}{6 n_{\mathrm{e}}^{3}}+I\left(n_{\mathrm{e}}\right)\right],
$$

is the so-called Sagdeev potential, and

$$
\begin{aligned}
I\left(n_{\mathrm{e}}\right)= & c_{0}+\frac{c_{1}+c_{2} n_{\mathrm{i}}}{n_{\mathrm{e}}}+\frac{c_{3}+c_{4} n_{\mathrm{i}}}{n_{\mathrm{e}}^{2}}+\frac{c_{5}+c_{6} n_{\mathrm{i}}}{n_{\mathrm{e}}^{3}} \\
& +c_{7} \ln \frac{\left(A_{\mathrm{i}}-N_{\mathrm{i} 0}\right) Z_{\mathrm{i}} n_{\mathrm{i}}-Z_{\mathrm{i}} n_{\mathrm{i} 0} n_{\mathrm{e}}}{N_{\mathrm{i} 0}\left(1-Z_{\mathrm{i}} n_{\mathrm{i}} 0\right) n_{\mathrm{e}}}
\end{aligned}
$$

is a new modification term for the Sagdeev potential $K\left(n_{\mathrm{e}}\right)$ in the presence of the second ion species with density $n_{\mathrm{i}}$

$$
\begin{aligned}
n_{\mathrm{i}}= & \frac{\sqrt{\left[\left(A_{\mathrm{i}}-1\right) n_{\mathrm{e}}+N_{\mathrm{i} 0}-A_{\mathrm{i}}\right]^{2}+4 N_{\mathrm{i} 0} n_{\mathrm{e}}}}{2 Z_{\mathrm{i}}\left(A_{\mathrm{i}}-1\right)} \\
& +\frac{\left(A_{\mathrm{i}}-1\right) n_{\mathrm{e}}+N_{\mathrm{i} 0}-A_{\mathrm{i}}}{2 Z_{\mathrm{i}}\left(A_{\mathrm{i}}-1\right)},
\end{aligned}
$$

where the parameter $N_{\mathrm{i} 0}=\left(A_{\mathrm{i}}-1\right) Z_{\mathrm{i}} n_{\mathrm{i} 0}$ and $M_{z}=M / k_{z}$. Based on the existential criterion for a solution of Eq. (6) (Wu et al. 1996; Wu \& Chao 2004b), one has $K\left(n_{\mathrm{e}}\right)<0$ for $n_{\mathrm{e}}$ between 1 and $n_{\mathrm{e} m}$ and $K\left(n_{\mathrm{e}}\right)=0$ at $n_{\mathrm{e}}=1$ and $n_{\mathrm{e}}=n_{\mathrm{e} m}$. In particular, from Eq. (7) $K\left(n_{\mathrm{e} m}\right)=0$ gives us the so-called nonlinear dispersion relation as follows:

$M_{z}^{2}=\left(1+2 n_{\mathrm{e} m}^{-1}\right) / 3+2 n_{\mathrm{e} m}^{2}\left(1-n_{\mathrm{e} m}\right)^{-2} I\left(n_{\mathrm{e} m}\right)$

From Eq. (7), one can find that the Sagdeev potential $K\left(n_{\mathrm{e}}\right)$ restores its form in an electron-proton plasma when $n_{\mathrm{i} 0}=n_{\mathrm{i}}=0$, hence the ion modified term $I\left(n_{\mathrm{e}}\right)=0$ (Wu et al. 1995). The constant coefficients $c_{0}, c_{1}, \ldots, c_{7}$ in the ion modified term $I\left(n_{\mathrm{e}}\right)$ are functions of the ion parameters $A_{\mathrm{i}}, Z_{\mathrm{i}}$, and $n_{\mathrm{i} 0}$.

Without loss of generality, we take one-time ionized oxygen $\mathrm{O}^{+}$as a special example of heavy ions. For $\mathrm{O}^{+}$, the parameters $A_{\mathrm{i}}=16$ and $Z_{\mathrm{i}}=1$ and the Sagdeev potential $K\left(n_{\mathrm{e}}\right)$ versus $n_{\mathrm{e}}$ is plotted in Fig. 1 for the cases of the background ion abundance ratio $n_{\mathrm{i} 0}=0.1,0.5$, and 0.8 , and the extremum $n_{\mathrm{e} m}=0.5$ has been used. From Fig. 1, the wave also can be accompanied by a dip density soliton in the three-component plasma with the low- $\beta$ of $\alpha \ll 1$, similar with that in an electronproton plasma (Wu et al. 1995, 1996). Figure 2 shows the parallel phase speed $M_{z}$ of the wave versus the density amplitude, $\delta n_{\mathrm{e} m} \equiv 1-n_{\mathrm{e} m}$ for the cases of the perturbed ion abundance ratio $n_{\mathrm{i} 0}=0.1,0.5$, and 0.8. From Fig. 2, one can see that the higher ion abundance ratio causes the wave to propagate more slowly because the higher ion abundance ratio implies that the plasma is more massive.

\section{Energization of heavy ions in nonlinear KAW}

In the field-aligned direction, the energization of charged particles is caused simply by direct acceleration of the parallel electric field $E_{z}$ so that $m_{\mathrm{s}} v_{\|}^{2} / 2=\left|q \phi_{z}\right|$; that is, the field-aligned energization depends on the field-aligned electric potential of the nonlinear KAW $\phi_{z}$ and on the particle charge $q$, but free of its mass. On the other hand, in the cross-field, the particle cannot be accelerated directly by the perpendicular field $E_{x}$ because of its Lamor cyclotron motion. In consequence, the cross-field energization depends on its gyrofrequency, hence on its mass. The 


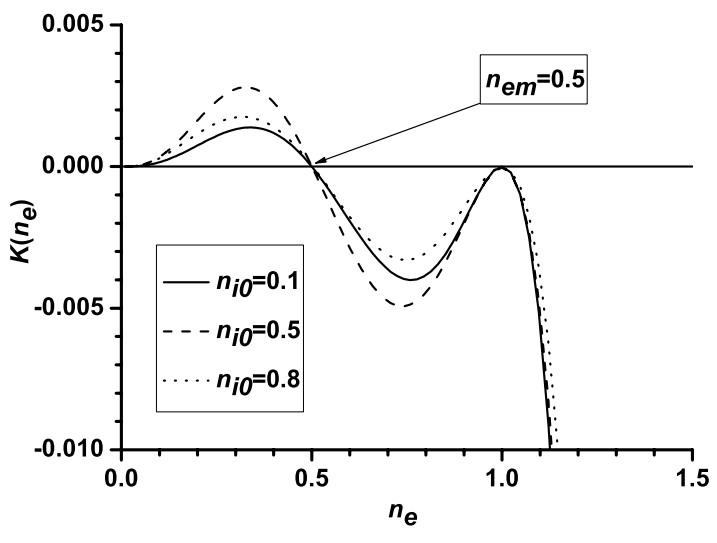

Fig. 1. The Sagdeev potential $K\left(n_{\mathrm{e}}\right)$ : solid, dashed, and dotted lines represent the cases of the background ion abundance $n_{\mathrm{i} 0}=0.1,0.5$, and 0.8 , respectively, and the amplitude of electron density $\delta n_{\mathrm{em}}=0.5$ has been used.

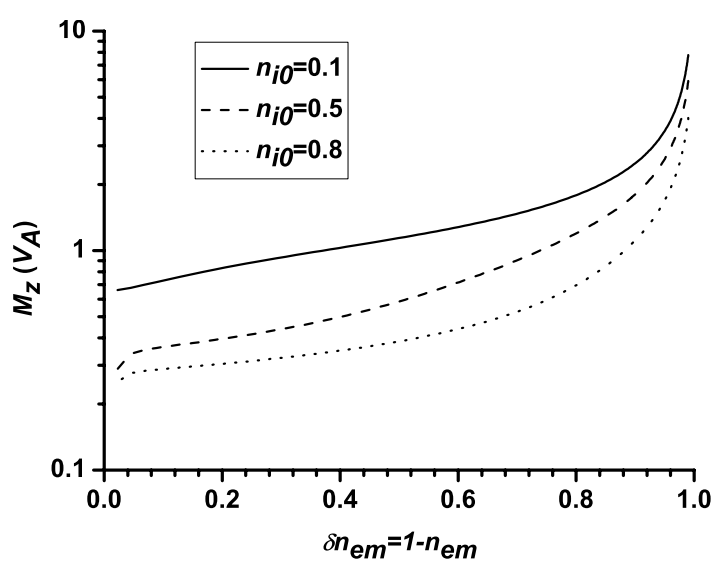

Fig. 2. The parallel phase speed $M_{z}$ : solid, dashed, and dotted lines represent the cases of the background ion abundance $n_{\mathrm{i} 0}=0.1,0.5$, and 0.8 , respectively.

field-aligned and cross-field velocities of particles in the KAW can be obtained from Eq. (1) as follows:

$v_{\mathrm{e} z}=\left(1-n_{\mathrm{e}}^{-1}\right) M_{z}$,

$v_{\mathrm{p} x}=\left(1-n_{\mathrm{p} 0} n_{\mathrm{p}}^{-1}\right) M_{z} \cot \theta$,

$v_{\mathrm{i} x}=\left(1-n_{\mathrm{i} 0} n_{\mathrm{i}}^{-1}\right) M_{z} \cot \theta$,

where the boundary conditions $v_{\mathrm{e} z}, v_{\mathrm{p} x}, v_{\mathrm{i} x}=0, n_{\mathrm{e}}=1, n_{\mathrm{p}}=n_{\mathrm{p} 0}$, $n_{\mathrm{i}}=n_{\mathrm{i} 0}$ for $\eta \rightarrow \infty$ have been used, and the ion density $n_{\mathrm{i}}$ is given by Eq. (9). This indicates that electrons move mainly along the magnetic field because of the smallness of their gyroradius and that the cross-field motions of protons and ions are driven by the electric polarization drift in the KAW and depend on the mass-charge ratio $A_{\mathrm{i}}$. In fact, from Eq. (3), one has $v_{\mathrm{i} x} / v_{\mathrm{p} x}=A_{\mathrm{i}}$; that is, the ion cross-field velocity is directly proportional to its mass-charge ratio. Figure 3 clearly shows this velocity relationship, where the maximal value of the ion cross-field velocity in the KAW, $v_{\mathrm{ixm}}$, is higher than that of protons, $v_{\mathrm{p} x m}$, by a factor $\sim 16$ for the one-time ionized oxygen. It also shows the maximal value of the electron field-aligned velocity, $v_{\mathrm{e} z m}$. For the five-time ionized oxygen that is the case in the corona, one likewise has $v_{\mathrm{i} x \mathrm{~m}} / v_{\mathrm{p} x \mathrm{~m}}=3.2$.

From Fig. 3, one can find that the proton cross-field velocity is lower than the electron field-aligned velocity by a factor of $\tan \theta$ in the case of a low ion abundance ratio of $n_{\mathrm{i} 0}<10 \%$,

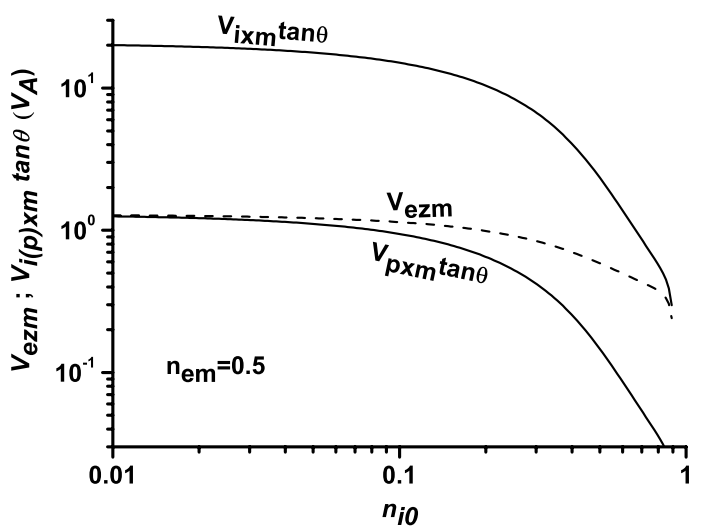

Fig. 3. Plots of the maximal velocities of electrons, protons, and ions in the wave versus the background ion abundance ratio $n_{\mathrm{i} 0}$, where the velocities of protons and ions have been multiplied by a factor of $\tan \theta$.

where $\theta$ is the KAW propagating angle. As a qualitative estimation, let us consider the quasi-perpendicular propagating case of the KAW in a low- $\beta$ plasma, where $\tan \theta \sim 1 / \sqrt{Q}(\mathrm{Wu}$ $\&$ Chao 2004b). This gives us the following velocity relation: $v_{\mathrm{i} x m} \sim A_{\mathrm{i}} v_{\mathrm{p} x m} \sim A_{\mathrm{i}} \sqrt{Q} v_{\mathrm{e} z m}$. In consideration of the smallness of the perpendicular scale of KAWs, we can imagine that these localized nonlinear structures of KAWs distribute randomly in the magnetized plasma. In particular, their moving directions in the perpendicular plane (i.e. the $k_{x}$ axis) are random such that the motion of the ions energized by them manifests itself as a random motion or turbulence. This indicates that the heavy ions have an effective perpendicular temperature $T_{\mathrm{i} \perp} \sim m_{\mathrm{i}} v_{\mathrm{i} x m}^{2} \sim$ $A_{\mathrm{i}}^{2}\left(m_{\mathrm{i}} / m_{\mathrm{p}}\right) m_{\mathrm{e}} v_{\mathrm{e} z m}^{2} \sim A_{\mathrm{i}}^{2}\left(m_{\mathrm{i}} / m_{\mathrm{p}}\right) T_{\mathrm{e} \|}$. On the other hand, according to the above scenario for the particle energization in the KAW, the ion parallel energy (or i.e. temperature) $T_{\mathrm{i} \|} \sim Z_{\mathrm{i}} T_{\mathrm{p} \|} \sim Z_{\mathrm{i}} T_{\mathrm{e} \|}$. In consequence, the ion-to-proton temperature ratio and the ion temperature anisotropy can be estimated by, respectively,

$T_{\mathrm{i}} / T_{\mathrm{p}} \sim A_{\mathrm{i}}^{2}\left(m_{\mathrm{i}} / m_{\mathrm{p}}\right)$

and

$T_{\mathrm{i} \perp} / T_{\mathrm{i} \|} \sim A_{\mathrm{i}}^{2}\left(m_{\mathrm{i}} / m_{\mathrm{p}}\right) Z_{\mathrm{i}}^{-1} \sim A_{\mathrm{i}}^{3} ;$

that is, the ion-proton temperature ratio is proportional to its mass number and the square of its mass-charge ratio and the ion temperature anisotropy to the cube of its mass-charge ratio. From Fig. 3 it can be found that the particle velocities in the KAW all decrease rapidly with the ion abundance ratio because the plasma becomes more and more massive with the increase in the ion abundance ratio.

The KAWs have been extensively observed by satellites (e.g. Freja, FAST, Polar, Cluster) in space plasmas (Louarn et al. 1994; Chaston et al. 1999; Stasiewicz et al. 2000) and have been shown to be in close relationship with the particle energization of the auroral plasma (Wygant et al. 2002; Chaston et al. 2003, 2005). Alfvén waves are one of most popular low-frequency electromagnetic fluctuations in the lower solar atmosphere and can be converted into KAWs when propagating outward due to the inhomogeneity, the parameter instability, or the nonlinear wave-wave coupling (see e.g. Shukla \& Sharma 2001; Shukla et al. 2004; Voitenko et al. 2003; Voitenko \& Goossens 2004b), although they cannot be observed directly in the current techniques of astronomic observations. We suggest they are what produce the preferential heating and temperature anisotropy of heavy ions observed by UVCS/SOHO in the corona (Kohl et al. 1997; Li et al. 1998; Esser et al. 1999; Cranmer et al. 1999; Marsch \& Tu 2001). 


\section{Summary and discussions}

In this letter we have investigated the nonlinear interaction of heavy ions with KAWs based on the three-component plasma model. It is shown that the ion energization is mass-dependent and anisotropic in which the ion cross-field energy is proportional to its mass and the square of its mass-charge ratio and the anisotropy (i.e. the perpendicular-parallel temperature ratio) to the cube of its mass-charge ratio.

As a special example, we took one-time ionized oxygen as the heavy ion species and estimated the ion-to-proton temperature ratio and the ion temperature anisotropy. It is straightforward to generalize these results to other cases including other ion species, such as $\mathrm{He}^{+}, \mathrm{O}^{5+}, \mathrm{Mg}^{9+}$, and so on. For example, the temperature ratio of $\mathrm{O}^{5+}$ to proton $T_{\mathrm{O}^{5+}} / T_{\mathrm{p}} \sim$ $\left(m_{\mathrm{O}} / m_{\mathrm{p}}\right)\left(m_{\mathrm{O}} / 5 m_{\mathrm{p}}\right)^{2} \sim 16 \times(16 / 5)^{2} \sim 160$, that is, the $\mathrm{O}^{5+}$ temperature $T_{\mathrm{O}^{5+}} \sim 1.6 \times 10^{8} \mathrm{~K}$ for the proton temperature of $T_{\mathrm{p}} \sim$ $10^{6} \mathrm{~K}$, and the temperature anisotropy of $\mathrm{O}^{5+}, T_{\mathrm{O}^{5+}} / T_{\mathrm{O}^{5+} \|} \sim$ $\left(m_{\mathrm{O}} / 5 m_{\mathrm{p}}\right)^{3} \sim 30$. These showed satisfy the requirement of the high ion cross-field temperatures (like $\mathrm{O}^{5+}$ and $\mathrm{Mg}^{9+}$ ) observed by UVCS/SOHO in the corona.

We anticipate that the results presented in this letter will help us understand the microphysics of the anisotropic and massdependent energization of the minor heavy ions observed recently in the corona.

Acknowledgements. This project has been supported by the NSFC under Grants No. $10425312,10373026,40574065$, and 10333030

\section{References}

Chaston, C. C., Carlson, C. W., Peria, W. J., Ergun, R. E., \& McFadden, J. P. 1999, Geophys. Res. Lett., 26, 647

Chaston, C. C., Bonnell, J. W., Carlson, C. W., et al. 2003, J. Geophys. Res., 108, 8003 Chaston, C. C., Bonnell, J. W., Carlson, C. W., et al. 2004, J. Geophys. Res., 109, A04205 Chaston, C. C., Peticolas, L. M., Carlson, C. W., et al. 2005, J. Geophys. Res., 110, A02211 Cranmer, S. R., Field, G. B., \& Kohl, J. L. 1999, ApJ, 518, 937

Esser, R., Fineschi, S., Dobrzycka, D., et al. 1999, ApJ, 510, L63

Hasegawa, A., \& Chen, L. 1975, Phys. Rev. Lett., 35, 370

Hasegawa, A., \& Uberoi, C. 1982, The Alfvén Wave, DOE Critical Review Series Advances in Fusion Science and Engineering (Technical Information Center, DOE, Washington)

Hollweg, J. V., \& Isenberg, P. A. 2002, J. Geophys. Res., 107, 1147

Hong, X., Ofman, L., \& Viñas, A. 2004, J. Geophys. Res., 109, A08103

Kletzing, C. A., et al. 2003, Phys. Rev. Lett., 90, 035004

Kohl, J. L., Noci, G., Antonucci, E., et al. 1997, Sol. Phys., 175, 613

Leneman, D., Gekelman, W., \& Maggs, J. 1999, Phys. Rev. Lett., 82, 2673

Li, X., Habbal, S. R., Kohl, J., \& Noci, G. 1998, ApJ, 501, L133

Louarn, P., Wahlund, J. E., Chust, T., et al. 1994, Geophys. Res. Lett., 21, 1847

Marsch, E., \& Tu, C. Y. 2001, J. Geophys. Res., 106, 227

Ofman, L., Gary, S. P., \& Viñas, A. 2002, J. Geophys. Res., 107, 1461

Shukla, A., \& Sharma, R. P. 2001, Phys. Plasmas, 8, 3759

Shukla, A., Sharma, R. P., \& Malik, M. 2004, Phys. Plasmas, 11, 2068

Stasiewicz, K., Bellan, P., Chaston, C., et al. 2000, Space Sci. Rev., 92, 423

Voitenko, Y., Goossens, M., Sirenko, O., \& Chian, A. C.-L. 2003, A\&A, 409, 331

Voitenko, Y., \& Goossens, M. 2004a, ApJ, 605, L149

Voitenko, Y., \& Goossens, M. 2004b, Nonl. Proc. Geophys., 11, 535

Voitenko, Y., \& Goossens, M. 2005a, Phys. Rev. Lett., 94, 135003

Voitenko, Y., \& Goossens, M. 2005b, J. Geophys. Res., 110, A10S01

Wu, D. J., \& Chao, J. K. 2003, Phys. Plasmas, 10, 3787

Wu, D. J., \& Chao, J. K. 2004a, J. Geophys. Res., 109, A06211

Wu, D. J., \& Chao, J. K. 2004b, Nonl. Proc. Geophys., 11, 631

Wu, D. J., \& Fang, C. 1999, ApJ, 511, 958

Wu, D. J., \& Fang, C. 2003, ApJ, 596, 656

Wu, D. J., Wang, D. Y., \& Fälthammar, C. G. 1995, Phys. Plasmas, 2, 4476

Wu, D. J., Huang, G. L., Wang, D. Y., \& Fälthammar, C. G. 1996, Phys. Plasmas, 3, 2879

Wygant, J. R., Keiling, A., Cattell, C. A., et al. 2002, J. Geophys. Res., 107, 1201

Yang, L., \& Wu, D. J. 2005, Phys. Plasmas, 12, 062903 